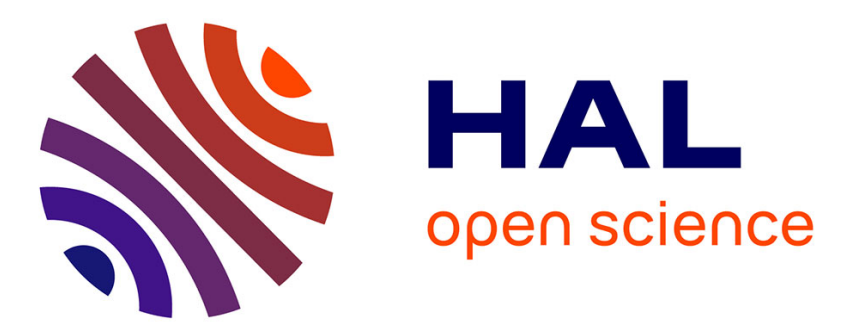

\title{
NMOS Device Optimization for the Design of a W-band Double-Balanced Resistive Mixer
}

\author{
Christophe Viallon, Grégory Ménéghin, Thierry Parra
}

\section{To cite this version:}

Christophe Viallon, Grégory Ménéghin, Thierry Parra. NMOS Device Optimization for the Design of a W-band Double-Balanced Resistive Mixer. IEEE Microwave and Wireless Components Letters, 2014, 24 (9), pp. 637-639. 10.1109/LMWC.2014.2332100 . hal-01015086

\section{HAL Id: hal-01015086 \\ https://hal.science/hal-01015086}

Submitted on 25 Jun 2014

HAL is a multi-disciplinary open access archive for the deposit and dissemination of scientific research documents, whether they are published or not. The documents may come from teaching and research institutions in France or abroad, or from public or private research centers.
L'archive ouverte pluridisciplinaire HAL, est destinée au dépôt et à la diffusion de documents scientifiques de niveau recherche, publiés ou non, émanant des établissements d'enseignement et de recherche français ou étrangers, des laboratoires publics ou privés. 


\title{
NMOS Device Optimization for the Design of a W-band Double-Balanced Resistive Mixer
}

\author{
Christophe Viallon, Member, IEEE, Grégory Ménéghin, and Thierry Parra
}

\begin{abstract}
This letter describes the implementation of NMOS devices in a passive ring mixer whose operating frequency reaches device's cut-off frequency. Conversion gain, linearity and required LO power are discussed regarding device geometry using simple analytic formulas and electrical simulations. The mixer is then embedded in a down-converter including RF, $L O$ and IF buffers and integrated in a $130 \mathrm{~nm}$ BiCMOS SiGe technology. Measurements indicate a conversion gain of $14.5 \mathrm{~dB}$ at $76.8 \mathrm{GHz}$, an output-referred $1 \mathrm{~dB}$ compression point of $-10 \mathrm{dBm}$ and a DSB noise figure of $6.3 \mathrm{~dB}$ confirming the interest of double-balanced passive mixers at millimeter-wave frequencies.
\end{abstract}

Index Terms-millimeter-wave, low-noise, resistive mixer, passive mixer, W-band, zero-IF receiver.

\section{INTRODUCTION}

$\mathbf{T}$ HE last decade has seen a growing number of applications in the millimeter-wave frequency range. Under constant developments, nanoscale silicon technologies become competitive with III-V for applications exceeding $100 \mathrm{GHz}$.

Whereas resistive mixers are widely used on III-V technologies at such high frequencies, active mixer topologies seems to be so far preferred on Si technologies. W-band active mixers meet an abundant literature [1]-[3]. These mixers provide good port-to-port isolation and conversion gain. Nevertheless, some recent works have reported $60 \mathrm{GHz}$ single-ended [4] [5] and single-balanced [6] resistive mixers using 90, 130 and $65 \mathrm{~nm}$ CMOS process respectively, and more recently, a $65 \mathrm{~nm}$ CMOS single-balanced resistive mixer has been demonstrated at $283 \mathrm{GHz}$ in a sub-harmonic configuration [7]. Despite conversion losses, these resistive mixer topologies exhibit better linearity and better noise performances, particularly for zeroIF receivers because the absence of flicker noise.

In this letter, a double-balanced resistive mixer is demonstrated in a $130 \mathrm{~nm}$ BiCMOS SiGe process. As presented in section II, mixer performances can be kept at frequencies close to NMOS cut-off frequency $\left(f_{t} \simeq 85 \mathrm{GHz}\right.$ ), when device geometry is carefully optimized. The implementation of this mixer within a zero-IF UWB automotive radar down-converter, as well as overall performances are given, then, in section III.

Manuscript received September 27, 2013; revised February 12, 2014 and April 25, 2014; accepted June 11, 2014. This work was supported under the ANR funded project VeLO and STMicroelectronics, Crolles, France.

C. Viallon and T. Parra are with LAAS-CNRS and Univ. de Toulouse, UPS, LAAS, 7 avenue du colonel Roche, F-31031 Toulouse, France (email: cviallon@laas.fr).

G. Ménéghin was with the LAAS-CNRS and is now with Exem, Labège, F-31670, France.

\section{Design Methodology}

A MOS device used under cold bias condition $\left(V_{D S}=0\right)$ acts as a simple conductance $g_{c h}$ whose value is controlled by the intrinsic part of Gate-Source Voltage $V_{g}$ (see Fig.1a).

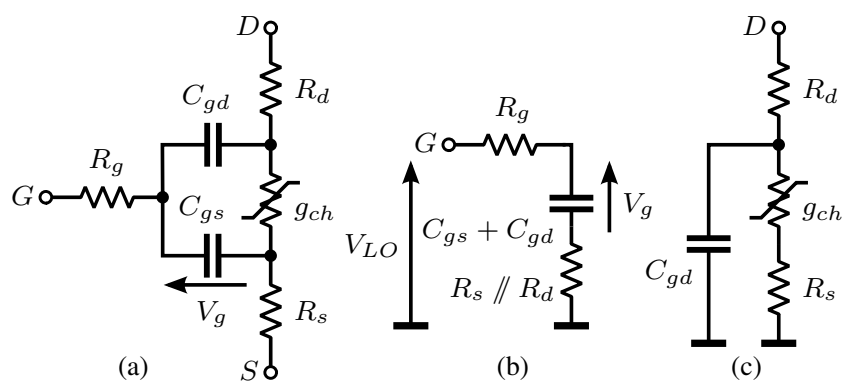

Fig. 1. Simplified equivalent circuit of a cold MOS device (a), ring mixer circuit seen by LO source (b), simplified Drain-Source impedance model (c).

A pump signal applied to the gate turns the channel into a time-varying nonlinear conductance that produces the frequency mixing. Two major limitations appear as the transistor is pushed toward its frequency limits.

The first one is located at the command side of the device, between gate and source connections. The channel conductance is controlled by the voltage $V_{g}$ across $C_{g s}$ (Fig.1a). The ratio between $V_{g}$ and $V_{G S}$ is decreasing as frequency increases and depends on the extrinsic elements $R_{g}$ and $R_{s}$. When a differential LO voltage is applied across the gates of the four mixing devices of a ring mixer, drains and sources appear to be virtually grounded at the LO frequency. Since $R_{d} \cong R_{s}$ and $C_{g s} \cong C_{g d}$ at $V_{D S}=0, V_{g}$ is deduced from $V_{L O}$ using the equivalent circuit of the LO input of the mixer (Fig.1b):

$$
V_{g} \simeq \frac{V_{L O}}{1+j \omega\left(R_{g}+R_{d} / / R_{s}\right)\left(C_{g s}+C_{g d}\right)}
$$

This expression suggests that the device remains efficiently driven as long as the condition $\omega_{L O} \ll \omega_{c 1}$ is fulfilled, with

$$
\omega_{c 1}=\frac{1}{\left(R_{g}+R_{d} / / R_{s}\right)\left(C_{g s}+C_{g d}\right)}
$$

$\omega_{c 1}$ is maximized using the smallest possible NMOS device, since $C_{g s}$ and $C_{g d}$ are proportional to gate area.

The second limitation comes from the drain-source timevarying equivalent impedance (Fig.1c). This network is derived from Fig.1 a given that for a ring mixer gates are virtually grounded at RF frequency and that $C_{g s}$ and $C_{g d}$ reactances are much greater than $R_{g}$ and $R_{s}$ [8]. The lowest and highest values taken by $g_{c h}$ are driven by the amplitude of $V_{g}$ and the 
DC bias. The drain-source impedance then swings between $Z_{o n}$ and $Z_{\text {off }}$ :

$$
\begin{gathered}
Z_{o n} \simeq R_{s}+R_{d}+\frac{1}{g_{c h}}=R_{s}+R_{d}+\frac{L}{W} \rho_{c h} \\
Z_{\text {off }} \simeq R_{d}+\frac{1}{j \omega C_{g d}} \simeq \frac{1}{j \omega C_{g d}}
\end{gathered}
$$

with $L, W$ the gate length and width, respectively, and $\rho_{c h}$ the channel resistivity. The equivalent circuit displayed on Fig.1c suggests that the transistor is operating as a diode and thus can be analyzed in the same way [9]. Hence, the optimal conversion losses $L_{\text {opt }}$ depend on the $Z_{\text {on }} / Z_{\text {off }}$ ratio:

$$
\begin{gathered}
L_{o p t}=1+2\left(\frac{Z_{o n}}{Z_{o f f}}\right)^{2}\left[1+\sqrt{1+\left(\frac{Z_{o n}}{Z_{o f f}}\right)^{2}}\right] \\
\left|\frac{Z_{o n}}{Z_{o f f}}\right| \simeq \frac{\omega_{R F}}{\omega_{c 2}} \text { with } \omega_{c 2} \simeq \frac{1}{\left(R_{s}+R_{d}+\frac{L}{W} \rho_{c h}\right) C_{g d}}
\end{gathered}
$$

$L_{o p t}$ is lowered if $\omega_{c 2}$ is maximized. As for $\omega_{c 1}$, this condition occurs using the smallest NMOS device. The resistive mixer is then theoretically optimized for low conversion losses but the linearity must be now investigated.

The linearity is mainly affected by the transition time between ON-state and OFF-state of NMOS devices which produce the mixing. This switching time may be reduced by improving the rate of change of the channel conductance $g_{D S}$ over $V_{G S}$. Starting from the simple square-law $I_{D S}$ expression of a NMOS device driven in triode region, the following relation is deduced [10]:

$$
\frac{\partial g_{D S}}{\partial V_{G S}}=\mu_{n} C_{o x} \frac{W}{L}
$$

with $\mu_{n}$ the electron mobility and $C_{O x}$ the gate-to-channel capacitance per unit area. This expression means that linearity is improved by increasing $W$. But parasitic effects are not taken into account and, as long as $W$ is increased, the aforementioned first limitation arises $\left(\omega_{c 1}\right)$, pulling down linearity.

One last remaining issue is the terminal resistances $R_{g}$, $R_{d}$ and $R_{s}$ of NMOS transistors. With today nanoscale MOS technologies, these resistance values appear to be quite large, especially $R_{g}$. As displayed on Fig.2, this resistance results from the contact resistance $R_{\text {con }}$ along with a salicided polysilicon resistance from contact to active region $R_{e x t}$, and the distributed gate resistance over active area [11], [12]. $R_{g}$ expression is then:

$$
R_{g}=\frac{R_{c o n}}{n}+\frac{R_{e x t}}{n}+\frac{R_{g} \square}{3} \frac{W_{f}}{n L}
$$

with $R_{g} \square$ the gate sheet resistance per square, $W_{f}$ the finger width, and $n$ the number of fingers. This expression points out that $R_{g}$ scales down as the number of gate fingers increases, as for $R_{s}$ and $R_{d}$ [13].

Finally, the expressions (2), (6) and (7) suggest that conversion losses and linearity of a resistive mixer are enhanced using NMOS devices with the smallest $L . W$ is chosen to provide the best linearity, and the number of fingers is increased to minimize the influence of extrinsic elements, because of (8). This result has been verified by performing electrical simulations on a resistive ring mixer fed with ideal baluns. The gates bias is adjusted near threshold voltage so that the best $Z_{\text {on }} / Z_{\text {off }}$ ratio can be reached from the swing of $V_{g}$. Conversion loss and output-referred $1 \mathrm{~dB}$ compression point $\left(O P_{1 d B}\right)$ are plotted on Fig. 3 at a $\mathrm{LO}$ power of $5 \mathrm{dBm}$ for various device geometries. As expected, the best conversion efficiency is obtained using a device with the smallest allowed length and width. The linearity is optimized by increasing $W$ up to the range $10-20 \mu \mathrm{m}$. Both characteristics are improved as the number of gate fingers is increased. However, no further improvement is observed above 8 fingers.

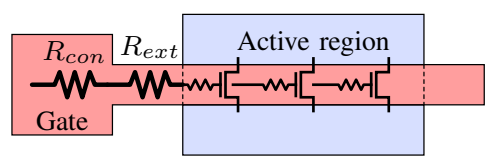

Fig. 2. Gate parasitic resistance distribution on MOS device.

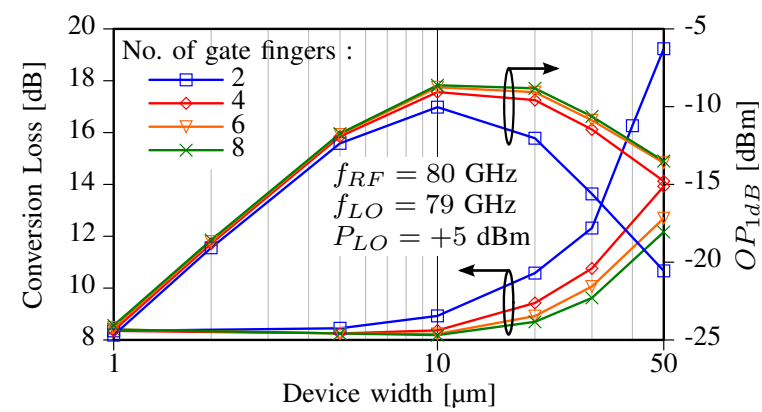

Fig. 3. Simulated conversion loss and $O P_{1 d B}$ of a ring mixer fed with ideal baluns for different NMOS width $W$ and number of fingers.

\section{DOWN-CONVERTER DESCRIPTION AND MEASUREMENTS}

Previous stated rules have been applied to the design of a double-balanced down-converter. The passive ring mixer using four $0.13 \times 2.5 \times 8 \mu \mathrm{m}^{2} \mathrm{NMOS}$ devices is surrounded by three amplifiers at RF, LO and IF terminals (Fig.4). SiGe HBTs are used for RF and LO buffers to take advantage of their attractive performances regarding noise and $f_{t}$. The total DC power consumption is $118 \mathrm{~mW}$ at $2.5 \mathrm{~V}(42 \mathrm{~mW}, 36 \mathrm{~mW}$ and $40 \mathrm{~mW}$ for LO, RF and IF buffers, respectively). More details on this chip are given in [14]. It has been fabricated using a $0.13 \mu \mathrm{m} \mathrm{SiGe} \mathrm{BiCMOS}$ process with HBT cutoff frequencies $f_{t} / f_{\max }$ of $230 / 280 \mathrm{GHz}$ from ST-Microelectronics. On-chip rat-race baluns have been included at RF and LO balanced terminals to enable single-ended on-wafer characterization. Without baluns and pads, the active part of the chip is $700 \times 400 \mu \mathrm{m}^{2}$. A specific test-bench has been developed to extract the conversion gain, compression point and noise figure. IF signal is measured from one of both outputs, the other one is connected to an AC-coupled $50 \Omega$ load. All measurement data are referred to the pads of the chip.

The conversion gain versus $\mathrm{LO}$ power is shown in Fig.5 at a LO frequency of $76 \mathrm{GHz}$. The measured $O P_{1 d B}$ is $-10 \mathrm{dBm}$. This power is strongly limited by the IF amplifier which 


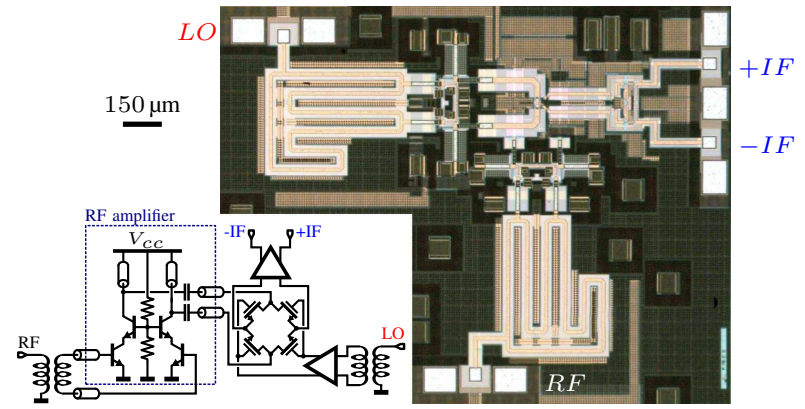

Fig. 4. Block-diagram and micro-photograph of the designed chip.

provides a gain of $\sim 14 \mathrm{~dB}$ from DC up to $2 \mathrm{GHz}$. It could be improved up to $+5 \mathrm{dBm}$ by increasing the linearity of the IF-amplifier since the simulated $O P_{1 d B}$ of the NMOS ring mixer, alone, is $-8 \mathrm{dBm}$ (Fig.3).

The double sideband noise figure has been extracted using the cold-source technique from measured conversion gains $G_{u s b}$ and $G_{l s b}$ at both upper and lower sideband around LO frequency. In our case, $G_{u s b}=G_{c}$. The noise figure $N F_{d s b}$ is extracted from the measured output noise power spectral density $N_{\text {out }}$ using:

$$
N F_{d s b}=\frac{N_{\text {out }}}{\left(G_{l s b}+G_{u s b}\right) k T_{0}}
$$

$k$ is Boltzmann's constant and $T_{0}$ is $290 \mathrm{~K}$. The noise figure of a resistive mixer can be close to conversion losses by minimizing $R_{s}$ and $R_{d}$. Moreover, this kind of mixer is well suited for zero-IF receiver because the absence of shot and flicker noises. Finally, the RF cascode amplifier acts as the RF transconductance stage in the Gilbert mixer: its gain and NF ( $\sim 9 \mathrm{~dB}$ and $\sim 5 \mathrm{~dB}$, respectively, under $50 \Omega$ input and output loads) decrease the NF of the overall down-converter.

The low-noise frequency conversion is confirmed by measurements of Fig.5, where $N F_{d s b}$ reaches a value of $6.3 \mathrm{~dB}$, for a conversion gain of $14.5 \mathrm{~dB}$, when the LO power exceeds $0 \mathrm{dBm}$. If we compare with all other published W-band mixers (Table I), these characteristics are quite attractive.

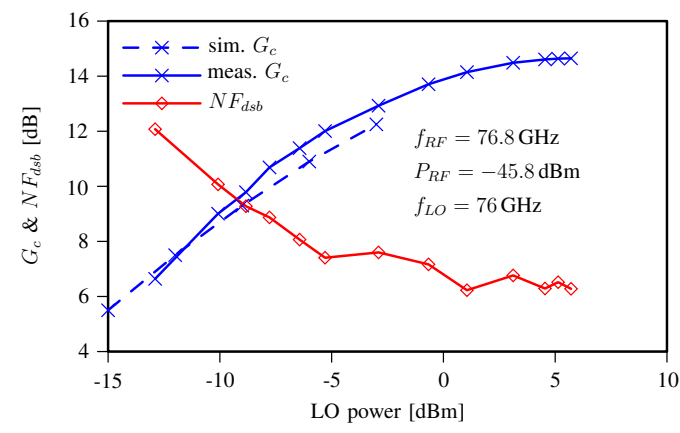

Fig. 5. Measured down-converter conversion gain and noise figure.

\section{CONCLUSION}

This letter investigates the optimization of a cold-biased NMOS resistive ring mixer. The geometry of NMOS transistor is widely discussed regarding conversion losses as well as
TABLE I

W-BAND MIXERS REPORTED WITH A LOW NF

\begin{tabular}{|l||l|l|l|l|}
\hline \multicolumn{1}{|l||}{ Ref. } & This work & \multicolumn{1}{|c|}{$[1]$} & {$[2]$} & {$[3]$} \\
\hline Technology & \multicolumn{3}{|c|}{$130 \mathrm{~nm}$ SiGe BiCMOS } & $\begin{array}{l}65 \mathrm{~nm} \\
\text { CMOS }\end{array}$ \\
\hline Mixer topology & Resistive & SB ${ }^{*}$ active & \multicolumn{2}{c|}{ Gilbert cell } \\
\hline$P_{D C}{ }^{* *}[\mathrm{~mW}]$ & 76 & N/A & 40 & 6 \\
\hline$f_{R F}[\mathrm{GHz}]$ & 76.8 & 77 & 73.8 & 76 \\
\hline$G_{c}[\mathrm{~dB}]$ & 14.5 & 20 & 8.5 & -1.5 \\
\hline$O P_{1 d B}[\mathrm{dBm}]$ & $-10^{* * *}$ & +4.3 & N/A & -9.5 \\
\hline$N F_{d s b}[\mathrm{~dB}]$ & 6.3 & 9.8 & 10.2 & 8.3 \\
\hline
\end{tabular}

${ }^{*}$ SB is for Single-Balanced, ${ }^{* *}$ LO buffer excluded, *** $+5 \mathrm{dBm}$ using optimized IF-amp.

linearity. Rules are derived that allow the mixer operation nearby the cut-off frequency of mixing devices. A doublebalanced resistive mixer is then demonstrated in the $77 \mathrm{GHz}$ range using a $0.13 \mu \mathrm{m}$ BiCMOS SiGe process. Simulated and measured results demonstrate that millimeter-wave Si-based resistive ring mixers can compete against their III-V-based equivalents in terms of linearity. In addition, a low noise figure is possible according to the measured $\mathrm{NF}$ of $6.3 \mathrm{~dB}$. To the authors' knowledge this result is the best ever reported at such high frequency using a Si-based technology.

\section{REFERENCES}

[1] S. K. Reynolds and J. D. Powell, "77 and 94-GHz downconversion mixers in SiGe BiCMOS," in Proc. IEEE Asian Solid-State Circuits Conf. (ASSCC), 2006, pp. 191-194.

[2] S. Nicolson et al., "A low-voltage SiGe BiCMOS 77-GHz automotive radar chipset," IEEE Trans. Microw. Theory Tech., vol. 56, no. 5, pp. 1092-1104, 2008.

[3] N. Zhang et al., "W-band active down-conversion mixer in bulk CMOS," IEEE Microw. Wireless Compon. Lett., vol. 19, no. 2, pp. 98-100, 2009.

[4] B. Motlagh et al., "Fully integrated $60-\mathrm{GHz}$ single-ended resistive mixer in 90-nm CMOS technology," IEEE Microw. Wireless Compon. Lett., vol. 16, no. 1, pp. 25-27, Jan. 2006.

[5] D. Jung and C. Park, "Cgs compensating v-band resistive mixer with low conversion loss at low LO power," Electron. Lett., vol. 46, no. 6, pp. 458-459, 2010.

[6] M. Varonen et al., "V-band balanced resistive mixer in 65-nm CMOS," in Proc. IEEE European Solid-State Circuits Conf. (ESSCIRC), 2007, pp. $360-363$.

[7] J. M. Guerra et al., "A $283 \mathrm{GHz}$ low power heterodyne receiver with on-chip local oscillator in $65 \mathrm{~nm}$ CMOS process," in IEEE Radio Freq. Integr. Circuits (RFIC) Symp. Dig., 2013, pp. 301-304.

[8] S. Maas, "A GaAs MESFET mixer with very low intermodulation," IEEE Trans. Microw. Theory Tech., vol. 35, no. 4, pp. 425-429, 1987.

[9] A. A. M. Saleh, Theory of Resistive Mixers. Cambridge, MA: M.I.T. Press, 1971.

[10] E. Lin and W. Ku, "Device considerations and modeling for the design of an InP-based MODFET millimeter-wave resistive mixer with superior conversion efficiency," IEEE Trans. Microw. Theory Tech., vol. 43, no. 8, pp. 1951-1959, 1995.

[11] J. J. Pekarik et al., "RF characterization and parameter extraction for CMOS device models," in Proc. IEEE Topical Meeting on Silicon Monolithic Integr. Circuits in RF Sys. (SiRF), 2007, pp. 36-39.

[12] X. Jin et al., "An effective gate resistance model for CMOS RF and noise modeling," in Int. Electron Devices Meeting (IEDM) Tech. Dig., 1998, pp. 961-964.

[13] S. Jen et al., "Accurate modeling and parameter extraction for MOS transistors valid up to $10 \mathrm{GHz}$," IEEE Trans. Electron Devices, vol. 46 , no. 11, pp. 2217-2227, 1999.

[14] G. Meneghin et al., "A double balanced resistive down-conversion mixer integrated in BiCMOS SiGe technology for $79 \mathrm{GHz}$ automotive radar," in Proc. IEEE NEWCAS-TAISA Conf., 2009, pp. 1-4. 